\title{
Positive Effect of Electroacupuncture Treatment on Gut Motility in Constipated Mice Is Related to Rebalancing the Gut Microbiota
}

\author{
Mingmin Xu $\left(\mathbb{D},{ }^{1}\right.$ Lu Wang $\left(\mathbb{D},{ }^{1}\right.$ Yu Guo $\mathbb{D}^{2},{ }^{2}$ Wei Zhang $\left(\mathbb{D},{ }^{3}\right.$ Ying Chen $\mathbb{D}^{1},{ }^{1}$ and Ying Li ${ }^{4}{ }^{4}$ \\ ${ }^{1}$ School of Acupuncture-Moxibustion and Tuina, Chengdu University of Traditional Chinese Medicine, Chengdu 610075, China \\ ${ }^{2}$ Teaching and Research Section of Acupuncture, School of Traditional Chinese Medicine, Jinan University, \\ Guangzhou 510632, China \\ ${ }^{3}$ Office of Educational Administration, Chengdu University of Traditional Chinese Medicine, Chengdu 610075, China \\ ${ }^{4}$ Graduate School, Chengdu University of Traditional Chinese Medicine, Chengdu 610075, China
}

Correspondence should be addressed to Ying Li; liying@cdutcm.edu.cn

Received 23 December 2020; Revised 13 February 2021; Accepted 23 February 2021; Published 3 March 2021

Academic Editor: Jing Hua Wang

Copyright ( $\odot 2021$ Mingmin Xu et al. This is an open access article distributed under the Creative Commons Attribution License, which permits unrestricted use, distribution, and reproduction in any medium, provided the original work is properly cited.

Functional constipation (FC) is a common and often recurrent functional bowel disorder that seriously affects the quality of life of affected individuals and incurs a significant economic burden on both the individual and society. There is accumulating evidence that intestinal dysbiosis contributes to constipation and that rebalancing the gut microbiota may be a novel therapeutic modality for FC. Electroacupuncture (EA) has been shown to restore the gut microbiota to normal levels in a variety of diseases. Additionally, several high-quality clinical studies have confirmed that EA is an effective, sustained, and safe treatment for FC. However, whether the effects of EA are secondary to changes in the gut microbiota and how EA modulates intestinal dysbiosis induced by constipation are unknown. Therefore, here, we focused on the potential regulatory mechanisms of EA on diphenoxylate-induced constipation in mice by analyzing structural changes in the gut microbiota. Our results showed that EA treatment effectively rebalanced the gut microbiota of constipated mice, mainly by decreasing the Firmicutes/Bacteroidetes ratio, which may represent one way in which EA promotes gastrointestinal motility and alleviates constipation. Our findings lay the foundation for further mechanistic and clinical research into the application of EA in patients with FC.

\section{Introduction}

Functional constipation (FC) is a common functional bowel disorder without identifiable structural or biochemical abnormalities [1]. According to the Rome IV criteria for functional gastrointestinal disorders, FC is defined as defecatory straining, hard or lumpy stools, a feeling of incomplete evacuation, defecatory obstruction, manual maneuvers to facilitate defecation, and fewer than three complete spontaneous bowel movements (CSBM) per week [2]. A 2020 epidemiological survey showed that the average prevalence of FC was $7.8 \%$ in three English-speaking Western countries (6.9\% in the United States, $7.9 \%$ in Canada, and $8.6 \%$ in the United Kingdom) [3]. In some patients, FC can be recurrent and refractory to treatment, resulting in significant pain and an impaired quality of life $[2,4]$. Clinically, FC can also result in abdominal distension, nausea, anorectal lesions, psychiatric symptoms, cardiovascular events, and even an increased risk of colorectal cancer $[2,5,6]$. Due to dietary and lifestyle changes, FC is affecting a growing number of people worldwide and incurring a consequent substantial economic burden on individuals and society $[4,7]$. For example, in the USA, FC accounts for over two million hospital visits and about 90 thousand hospitalizations annually, costing $\sim 7$ billion USD in diagnostic assessments $[4,7]$. Hence, prompt and aggressive treatment of constipation is an important strategy to prevent other diseases and reduce healthcare expenditures. Conventional treatments include medical therapies such as fiber, osmotic and stimulant laxatives, and selective 5-hydroxytryptamine receptor $4\left(5-\mathrm{HT}_{4}\right)$ agonists to relieve constipation when lifestyle modifications are ineffective [8]. However, nearly half of patients with FC are 
dissatisfied with current medical approaches due to a lack of response, drug dependence, and unpleasant side-effects [9]. As a result, patients are urgently seeking effective, safe, and nontoxic treatment alternatives.

Acupuncture, which originated in traditional Chinese medicine (TCM), has attracted considerable interest due to overwhelming clinical evidence that it is an effective and safe treatment for FC. TCM theory and practice propose that acupuncture, which is performed by inserting needles into specific points in the body and then stimulating the needles manually or electrically to elicit the Qi [10], can promote the intestinal function recovery [11]. Electroacupuncture (EA) is a modern acupuncture method that uses weak electrical stimulation of acupuncture needles instead of manual manipulation to enhance the acupuncture effect. As awareness of EA treatment grows, clinical studies have confirmed positive outcomes from EA therapy in the management of intestinal diseases such as irritable bowel syndrome [12] and quiescent inflammatory bowel disease [13] as well as other diseases such as stress urinary incontinence [14], migraine prophylaxis [15], insomnia [16], and postprandial distress syndrome [17]. Specifically related to bowel dysfunction, since 2019, several high-quality RCTs have confirmed that acupuncture can increase CSBM, soften stool hardness, alleviate defecation straining, reduce frequent rescue medicine use, reduce adverse events, and improve the quality of life of constipated patients $[18,19]$.

However, the mechanisms underlying EA for FC remain largely unknown. Intestinal dysbiosis contributes to constipation via several mechanisms, and accumulating evidence from fecal bacterial transplantation and microecological therapy indicates that chronic constipation can potentially be ameliorated by rebalancing the gut microbiota composition [20-22]. However, in a systematic review, the effects of probiotics on constipation remained uncertain [23]. Nevertheless, the curative effect of acupuncture on obesity and osteosarcoma has been shown to be related to restoring intestinal microflora to normal levels $[24,25]$, suggesting that intestinal dysbiosis disorders can be treated with acupuncture.

It is currently unknown whether acupuncture can modulate intestinal dysbiosis induced by constipation. We therefore hypothesized that the purgative effect of acupuncture is mediated by regulation of the gut microbiota. To test this hypothesis, we analyzed the effect of electroacupuncture (EA) on changes in the gut microbiota in diphenoxylate-induced constipation in mice [26-28].

\section{Materials and Methods}

2.1. Animals. Female Kunming mice weighing 20-25 g were purchased from Chengdu Dossy Experimental Animals Co., Ltd. (Chengdu, China), and group-housed (3-4 animals per cage) in individually ventilated cage systems. Animals were fed a standard commercial mouse chow and water ad libitum under a 12/12-hour light-dark cycle at a constant temperature $\left(25 \pm 2^{\circ} \mathrm{C}\right)$ and humidity $(60 \pm 10 \%)$.
2.2. Experimental Design. Twenty-four mice were randomly separated into a normal control (NC) group, slow transit constipation (STC) group, and STC treated with EA (STC.EA) group, with $n=8$ mice in each group. After one week of acclimation, STC was induced in the STC and STC.EA groups by intragastric administration of $10 \mathrm{mg} / \mathrm{kg}$ body weight diphenoxylate solution once a day for four consecutive weeks [26-28]; the NC group was administered an equivalent amount of saline. After four weeks of modeling, fecal parameters (the first black stool defecation time and feces number, weight, and water content) were measured to assess whether the STC models were successfully established. When every fecal parameter in STC and STC.EA mice showed significant differences compared with those in the NC group, the STC model was deemed successful. Then, mice in STC.EA group were treated daily with EA for two weeks, with two days of rest after five EA treatments. After the final treatment, fresh feces were collected from all mice in sterile tubes and then immediately frozen at $-80^{\circ} \mathrm{C}$ for $16 \mathrm{~S}$ rRNA sequencing. The day after fecal collection, fecal parameters were measured, and the day after that, intestinal transit rate was measured. Colons were excised and fixed in $4 \%$ paraformaldehyde for hematoxylin and eosin (HE) staining. The experimental protocol is shown in Figure 1.

2.3. EA Treatment. Mice were restrained in home-made mouse pockets before treatment to ease restraint stress, and mice in the NC and STC groups were also fixed in mouse pockets. Then, sterile acupuncture needles $(0.25 \times 13 \mathrm{~mm})$ were inserted vertically into the "Tianshu" (ST25) and "Shangjuxu" (ST37) unilaterally to a depth of 4-5 mm, and the left or right acupuncture points were alternately stimulated each time. The stainless steel acupuncture needles were connected to the Hwato SDZ-V electropulse acupuncture therapeutic apparatus (Hwato, Jiangsu, China) at a frequency of $3 / 15 \mathrm{~Hz}$ and an intensity of $1 \mathrm{~mA}$ for 30 minutes.

2.4. Measurement of Fecal Parameters. Mice were given $0.1 \mathrm{ml}$ of $5 \%$ activated carbon suspension after fasting for $12 \mathrm{~h}$ and then moved into individual empty cages lined with white blotting paper, where they received normal water and food. The length of time from activated carbon gavage to the first black stool defecation was recorded as the first black stool defecation time. The total fecal number and wet weight during a $6 \mathrm{~h}$ period were calculated. Each sample was dried in an oven at $90^{\circ} \mathrm{C}$ for $12 \mathrm{~h}$ and weighed. Fecal water content (\%) was measured by comparing the weight change before and after drying, where water content $(\%)=$ (wet weight - dry weight)/wet weight $\times 100 \%$ [29].

2.5. Measurement of Intestinal Transit Rate. All mice were fasted $12 \mathrm{~h}$ before measurement of intestinal transit rate and then orally administered activated carbon suspension. After a $30 \mathrm{~min}$ interval, mice were sacrificed by cervical dislocation, and the entire small intestine between the pyloric sphincter and ileocecal junction was excised and measured 


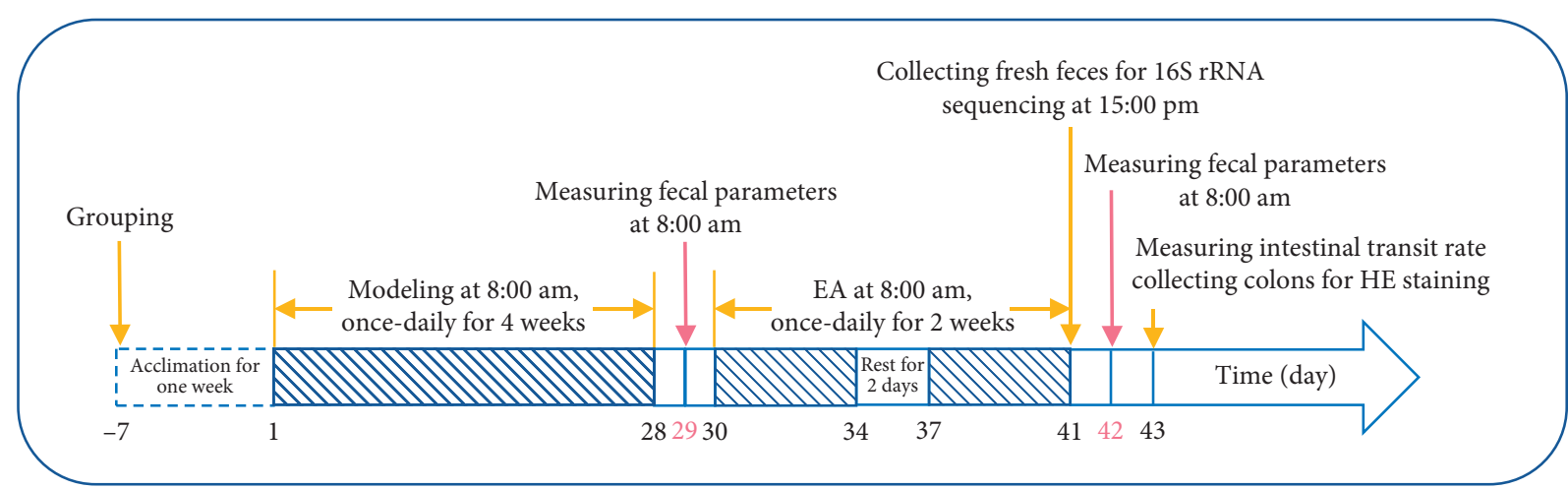

FIGURE 1: Experimental protocol. STC was induced in the STC and STC.EA groups by intragastric administration of diphenoxylate solution. Mice in the STC.EA group were treated with EA. In order to determine modeling success and the effect of EA on constipation, fecal parameters including the first black stool defecation time and feces number, weight, and water content were measured after the end of modeling (day 29) and EA treatment (day 42), respectively. The intestinal transit rate was measured on day 43 to assess the effect of EA on gut motility. The diversity and composition of fecal microbiota were detected by $16 \mathrm{~S}$ rRNA gene sequencing, and pathological changes in the colon were assessed by HE staining and histopathological examination. STC: slow transit constipation; EA: electroacupuncture; HE: hematoxylin and eosin.

[30]. The distance from the pyloric sphincter to the forefront of the activated carbon bulk was measured as the activated carbon migration distance, with the small intestinal transit rate (\%) calculated as the migration distance of activated carbon/overall length of small intestine $\times 100 \%$ [29].

2.6. $16 S$ rRNA Gene Sequencing and Analysis. All mouse gut microbe genomic DNA was extracted from fecal samples using the E.Z.N.A. Stool DNA Kit (Omega Bio-tek, Norcross, GA, USA) following the manufacturer's instructions under the required aseptic conditions. The quality, integrity, and concentration of each extracted DNA were determined by $1 \%$ agarose gel electrophoresis and optical density (OD) analysis with a NanoDrop ND-2000 spectrophotometer (Thermo Fisher Scientific, Waltham, MA, USA). The V3-V4 hypervariable region was amplified with a set of barcode primers (341F: $5^{\prime}$-CCTAYGGGRBGCASCAG- $3^{\prime}$ and 806R: $5^{\prime}$-GGACTACNNGGGTATCTAAT- ${ }^{\prime}$ ). Then, amplicons were extracted from $2 \%$ agarose gels, purified with the AxyPrep DNA Gel Extraction Kit (Axygen Biosciences, Union City, CA, USA), and quantified by QuantiFluor-ST (Promega, Madison, WI, USA). Purified amplicons were pooled in equimolar amounts and paired-end sequenced $(2 \times 250)$ on an Illumina HiSeq 2500 platform (Illumina, San Diego, CA, USA) according to standard protocols. The $16 \mathrm{~S}$ rRNA genes were sequenced by the Health Time Gene Institute, Shenzhen, China. The raw reads were deposited into the NCBI Sequence Read Archive (SRA) database (accession number: PRJNA688113).

2.7. Histological Analysis. Colons were fixed in $4 \%$ paraformaldehyde for $>48 \mathrm{~h}$. After washing with running water for $30 \mathrm{~min}$, colon tissue was dehydrated, vitrified, and then embedded in paraffin wax. Five-micron-thick sections were prepared using a Leica microtome (Wetzlar, Germany). After dewaxing, sections were stained with hematoxylin and eosin (HE) and dehydrated in graded alcohol and xylene.
The entire colonic section was scanned under a microscope to assess for histopathological changes and then photographed and recorded with a microscopic imaging system.

2.8. Bioinformatics and Statistical Analysis. After sequencing, data were processed with the QIIME toolkit (v1.9.1). Operational taxonomic units (OTUs) were clustered with an identity threshold of $97 \%$ sequence similarity using Uparse (v7.0.1001). Based on the results of normalized OTU abundance, the alpha diversity of microbes was calculated as the observed species, chao 1, Shannon index, and phylogenetic diversity (PD) whole tree to represent the diversity and richness of each sample. Subsequent statistical analyses were performed in R software (v3.5.2). Partial least squares discriminant analysis (PLS-DA) was used to reveal differences in OTU levels between samples. Metastat analysis was further used as a statistical method to detect significant differences in gut microbiome composition between groups based on Fisher's exact test and false discovery rate (FDR) calibration [31]. Linear discriminant analysis effect size (LEfSe) analysis was performed by using LEfSe software (v2.0) and the nonparametric rank-sum test. Spearman analysis was used to assess correlations. Fecal parameters and intestinal transit rate are presented as mean \pm standard deviation (SD) and analyzed with SPSS 21.0 software (IBM Statistics, Armonk, NY, USA). Multiple comparisons between groups were tested by independent one-way analysis of variance (ANOVA) with the least-significant difference (LSD) test. A $P$ value $<0.05$ was regarded as statistically significant.

\section{Results}

3.1. EA Treatment Significantly Improves Constipation and Promotes Gastrointestinal Motility. To investigate the efficacy of EA on constipation in mice, fecal parameters were measured. A mouse in the STC.EA group died during modeling, resulting in the following final sample sizes: NC 
group $(n=8)$, STC group $(n=8)$, and STC.EA group $(n=7)$. After four weeks of modeling, the fecal pellet number, wet weight, and fecal water contents were significantly lower, and the first black stool defecation time was significantly longer in STC and STC.EA mice compared with NC mice $(P<0.05$, Figures $2(\mathrm{a})-2(\mathrm{~d}))$, indicating that mice in the STC and STC.EA groups successfully modeled STC. There were no significant differences between the STC and STC.EA groups $(P>0.05)$.

After two weeks of EA treatment, fecal pellet number, wet weight, and fecal water contents were significantly lower, and the first black stool defecation time was significantly longer in STC compared with NC mice $(P<0.05$, Figures $2(\mathrm{a})-2(\mathrm{~d}))$. However, the symptoms of constipation were significantly improved in STC.EA compared to STC mice $(P<0.05$, Figures $2(\mathrm{a})-2(\mathrm{~d}))$. The intestinal transit rate was calculated to assess gastrointestinal motility and, compared with the NC group, the STC group had a significantly shorter intestinal transit rate $(P<0.05$, Figure $2(\mathrm{e}))$, but EA significantly increased the intestinal transit rate compared to the STC group $(P<0.05$, Figure $2(\mathrm{e}))$. In addition, histological assessment revealed that there were no significant differences in intestinal morphology between the three groups, as described in detail in the Supplementary Materials section, although isolated cases showed mild histopathological abnormalities (Figure S1). Taken together, these results indicate that EA markedly promotes gastrointestinal motility and improves the signs of constipation in mice.

\subsection{EA Treatment Reverses Constipation-Induced Structural} Changes in the Gut Microbiota. It has been reported that an imbalance in the intestinal flora can contribute to constipation. The three groups of mice produced 1088 OTUs in total (Figure 3(a)), and the rarefaction curves of fecal microbiota showed that the sequence depth was saturated (Figure 3(b)). To explore the effect of EA on gut microbiota richness and diversity, we analyzed the $\alpha$-diversity metrics of the three groups, but there were no significant differences between groups in terms of species, chao 1, Shannon index, and PD whole tree metrics $(P>0.05$, Fig. S2), reflecting that there was little change in gut microbiota diversity between groups. Subsequently, PLSDA was applied to compare specific differences in microbial composition between the three groups. The STC group was separated from the $\mathrm{NC}$ group by the first principal component (PC1), with the dominant species in the NC group including Christensenellaceae R-7group, Anaerofustis, Enterobacter, Ochrobactrum, Gelria, Ruminococcaceae TCG-014, and Hydrogenoanaerobacterium and the dominant species in the STC group including Ruminococcaceae TCG-009, Roseburia, and Candidatus Stoquefichus, suggesting that constipation may result in a gut microbiota disorder. The STC.EA group was separated from the STC group by the second principal component (PC2), with the gut microbiota of the STC.EA group exhibiting movement in PC1 towards the direction of the NC group, suggesting that STC-induced variations in the gut microbiota were reverted by EA treatment (Figure 3(c)).
3.3. EA Treatment Improves the Gut Microbiota at the Phylum Level in Constipated Mice. As illustrated in Figure 4(a), major phyla in all classes included Bacteroidetes and Firmicutes. Compared with the NC group, Firmicutes (46.6\%) and Proteobacteria (5.09\%) expanded at the expense of Bacteroidetes (47.4\%) in the STC group. Conversely, EA therapy decreased the abundance of Firmicutes (44.68\%) and Proteobacteria (2.04\%) and increased Bacteroidetes (51.75\%). The Firmicutes to Bacteroidetes ratio (F/B) is usually used to evaluate intestinal health. The F/B in the STC group was close to $1 / 1$, while the $\mathrm{F} / \mathrm{B}$ in the STC.EA group was similar to that in the NC group, indicating that EA treatment might rebalance the gut microbiota in constipated mice towards that seen in healthy mice (Figure 4(b)).

3.4. EA Treatment Improves Gut Microbiota at the Genus Level in Constipated Mice. The 35 most abundant genera in the three groups are shown in Figure 5(a). To further elucidate the main microbe genera altered after EA treatment, we used LEfSe and metastat analyses to examine differences in the relative abundance of gut microbiota at the genus level. LAD analysis showed that three genera (Roseburia, Turicibacter, and Lachnoclostridium) were dominant in the STC group (Figure 5(b)) but noticeably decreased in the STC.EA group. Metastat analysis demonstrated that Turicibacter, Lachnoclostridium, Ruminiclostridium 9, and Tyzzerella increased significantly in the STC group, while Muribaculum and Christensenellaceae $R-7$ decreased significantly $(P<0.05$, Table S1). Compared with the STC group, the STC.EA group had a significantly decreased relative abundance of Roseburia $(P<0.05$, Table $S 1)$. Further analysis of the aforementioned seven species in each group showed that EA treatment restored gut microbiota composition of constipated mice to levels similar to those seen in healthy mice (Figure 5(c)).

3.5. Correlation Analysis. As gut microbes interact with each other, correlations between certain microbial species selected by LEfSe and Metast analysis in the NC, STC, and STC.EA groups were calculated to investigate the effect of EA on these interactions. As shown in Figure 6, the interactions between microbes in the STC group were different from those in the NC group, especially microbes enriched in the STC group, which were reversed by EA treatment. For instance, correlations between g_Lachnoclostridium and c_Erysipelotrichia; o_Erysipelotrichales; f_Erysipelotrichaceae changed from a small negative correlation in the NC group to a significant positive correlation in the STC group to a smaller positive correlation in the STC.EA group. In addition, new correlations between g_Lachnoclostridium and g_Turicibacter emerged in the STC group but disappeared in the NC and STC.EA groups. These results suggest that EA treatment improved relationships between microbes disrupted by constipation in a beneficial way to maintain a healthy microbial community.

Furthermore, ChristensenellaceaeR-7, Muribaculum, Ruminiclostridium9, Tyzzerella, Turicibacter, Roseburia, and Lachnoclostridium were significantly correlated with 


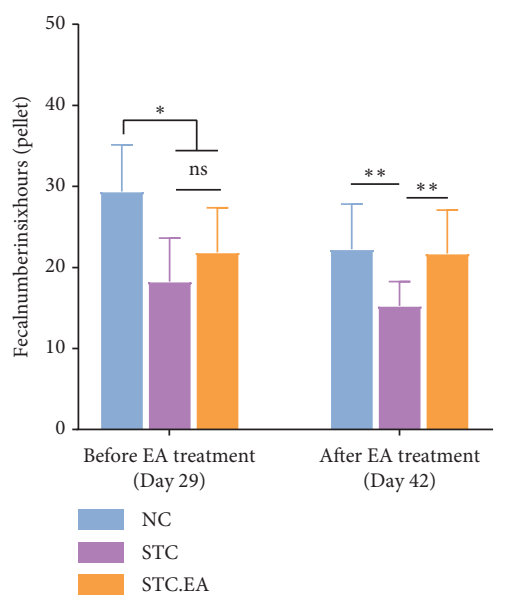

(a)

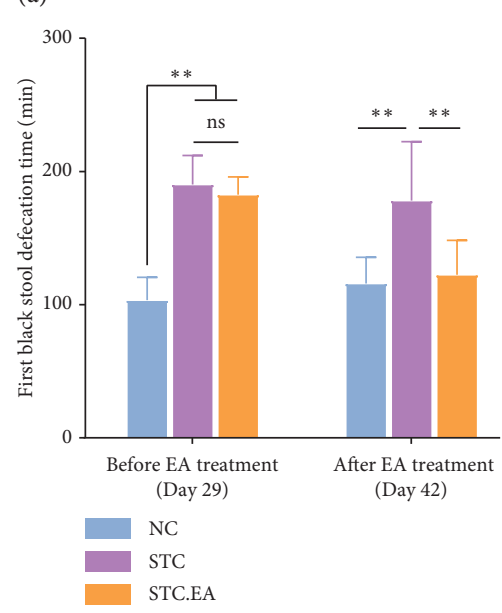

(d)

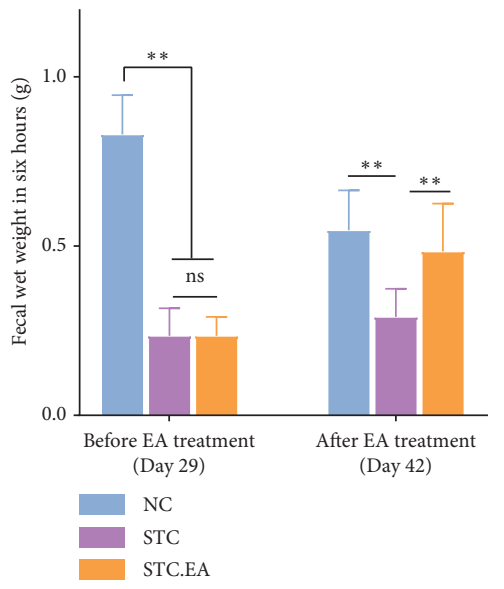

(b)

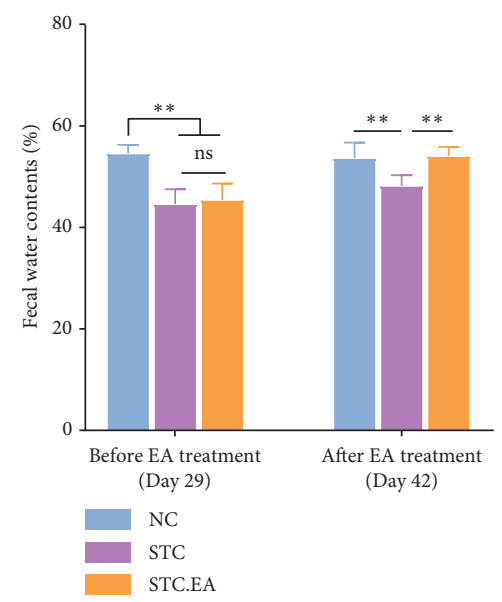

(c)

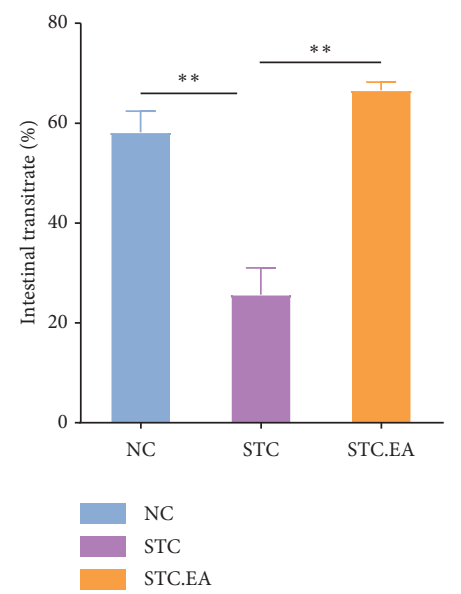

(e)

Figure 2: Effects of EA on the defecation status of constipated mice. (a) Fecal number. (b) Fecal wet weight. (c) Fecal water contents. (d) First black stool defecation time. (e) Intestinal transit rate. Fecal parameters (first black stool defecation time and fecal number, weight, and water contents) were measured before (day 29) and after (day 42) EA treatment. Data represent the mean \pm SD. ${ }^{*} P<0.05 ;{ }^{*}{ }^{*} P<0.01$.

constipation-related indices (Figure 7). In particular, Lachnoclostridium was negatively correlated with intestinal transit rate, fecal number, and wet weight while positively correlated with first black stool defecation time. Turicibacter was negatively correlated with intestinal transit rate, fecal water contents, and wet weight while positively correlated with first black stool defecation time.

\section{Discussion}

FC can be categorized into three different types, namely, normal transit constipation, slow transit constipation (STC), and defecatory or rectal evacuation disorders [8]. Among these, STC, characterized by a markedly increased gut transit time, is the most common type of FC. Recent and growing evidence illustrates the complicated interactions between intestinal dysbiosis and constipation, especially in STC [32-34]. Therefore, here, we used a murine STC model to evaluate the effectiveness of EA in relieving symptoms of constipation and its influence on the intestinal microbiota. Diphenoxylate acts directly on gastrointestinal smooth muscle cells to inhibit gut motility. We observed that diphenoxylate-induced constipated mice had typical symptoms of constipation, compromised gastrointestinal motility, and intestinal dysbiosis that closely corresponded to the clinicopathological features of STC.

EA is most commonly used in clinical trials and laboratory research, because it can be implemented uniformly by adjusting the current intensity and frequency. Thus, EA was chosen as the acupuncture modality to treat constipated mice in this study. Tianshu (ST25) and Shangjuxu (ST37) are the most commonly used acupoints for treating FC. Indeed, the largest published clinical trial of acupuncture for chronic severe FC confirmed that EA treatment at ST25 and ST37 increased CSBM and that its effect persisted for at least three months after treatment [18]. In animal experiments, EA applied to ST25 and ST37 also significantly improved constipation and gastrointestinal transit parameters $[35,36]$. In our study, STC mice receiving EA treatment at ST25 and ST37 had increased fecal number, wet weight, and water content, increased intestinal transit rate, and reduced first black stool defecation time, suggesting that EA effectively 

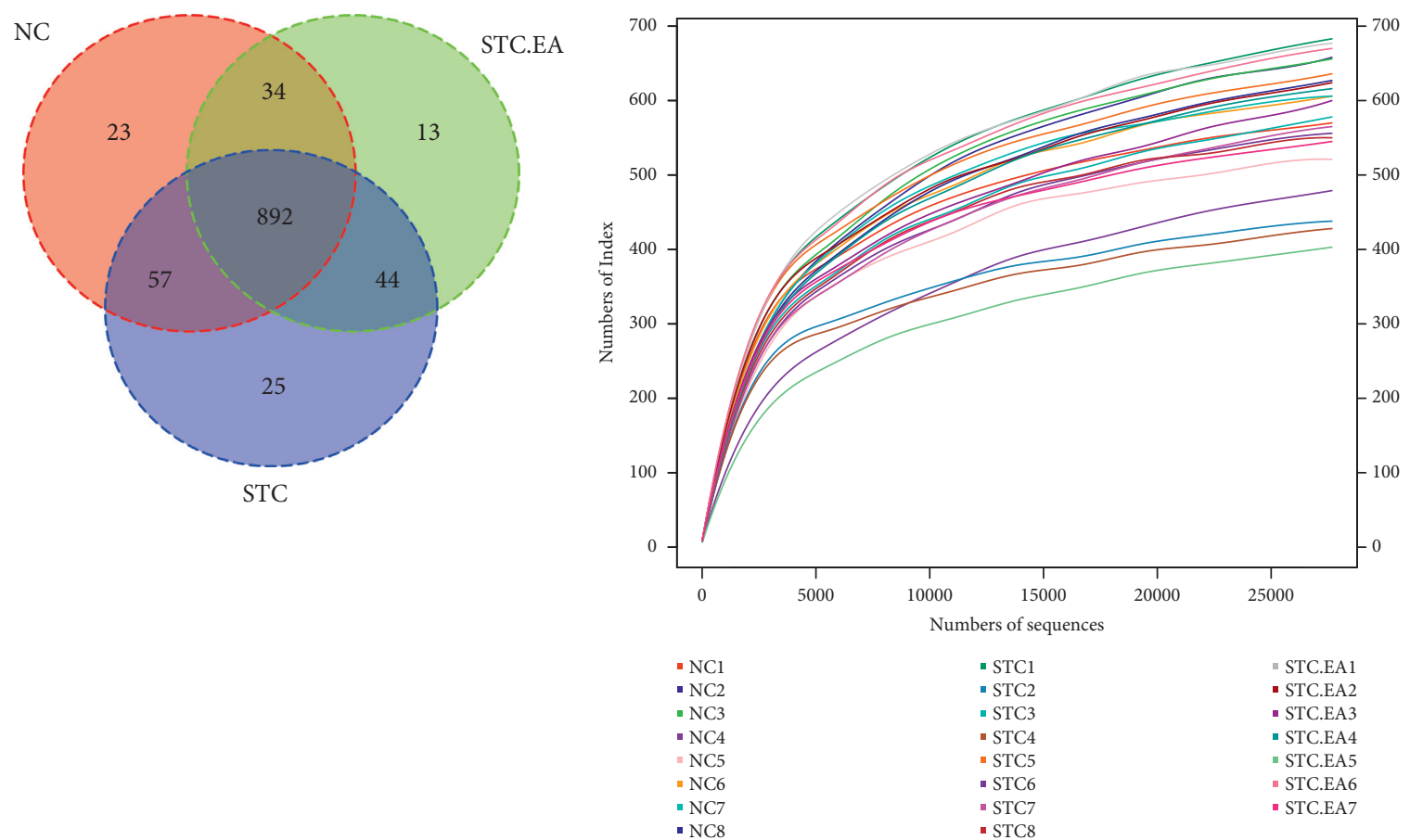

(a)

(b)

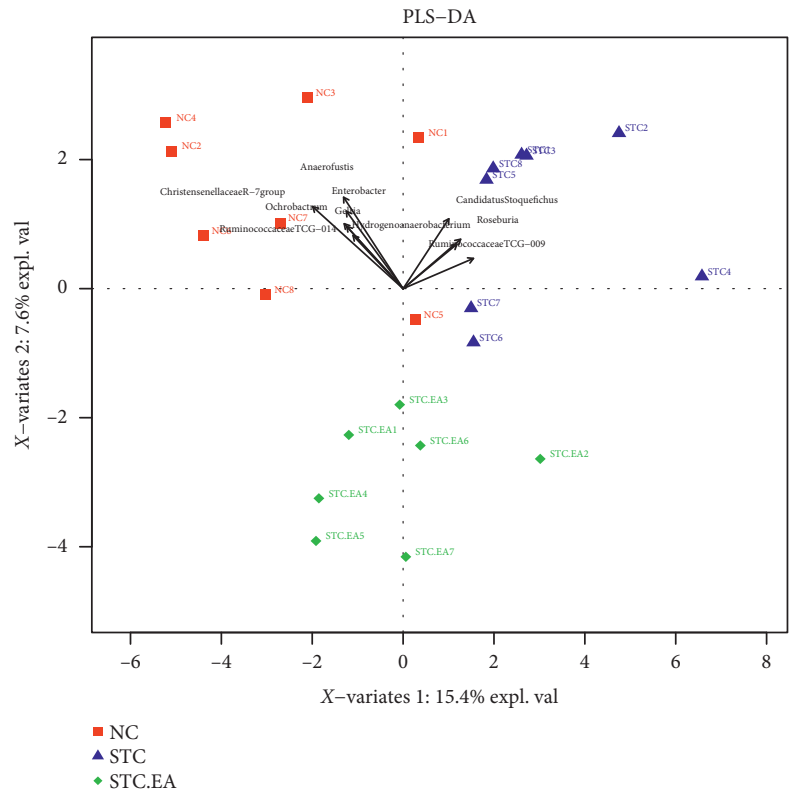

(c)

Figure 3: Effects of EA on the overall structure of the gut microbiota in constipated mice. (a) Venn diagram of shared and independent bacterial OTUs in the three groups. (b) Rarefaction curves using observed species index of OTU levels. (c) PLS-DA analysis at the OTU level.

accelerated gastrointestinal motility and alleviated constipation symptoms in this model. Zhu et al. reported that EA stimulation at ST37 had a positive therapeutic effect on constipation, most likely mediated by increasing 5-hydroxytryptamine and tryptophan hydroxylase levels in colonic tissues [37]. Liang et al. found that EA stimulation at either ST37 or ST25 relieved the symptoms of constipation but had different effects in the proximal colon. ST37 regulated both excitatory and inhibitory enteric neurons to normal levels, while ST25 only influenced inhibitory neurons [36]. Sato et al. and Noguchi et al. reported that EA treatment at specific acupoints influenced the sympathetic-parasympathetic balance to enhance gastrointestinal motility $[38,39]$. These studies partly explain how EA might treat FC, but several questions remain unanswered. Specifically, while EA treatment at ST25 or ST37 relieves constipation through different mechanisms, the mechanism of synergy of simultaneous EA stimulation at both ST36 and 


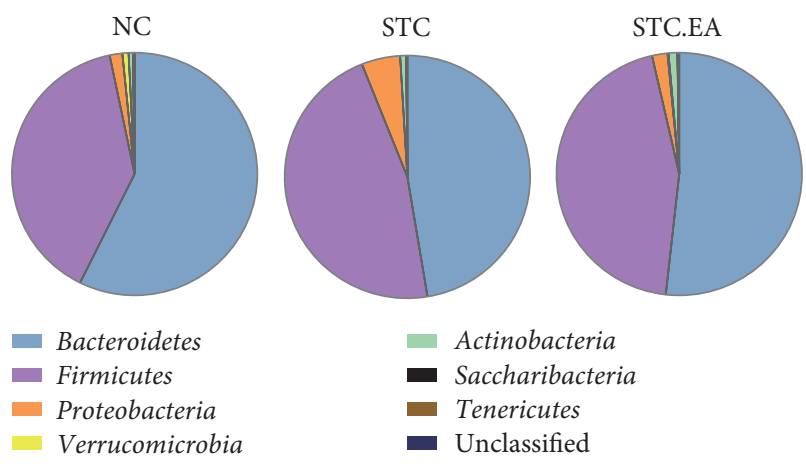

(a)

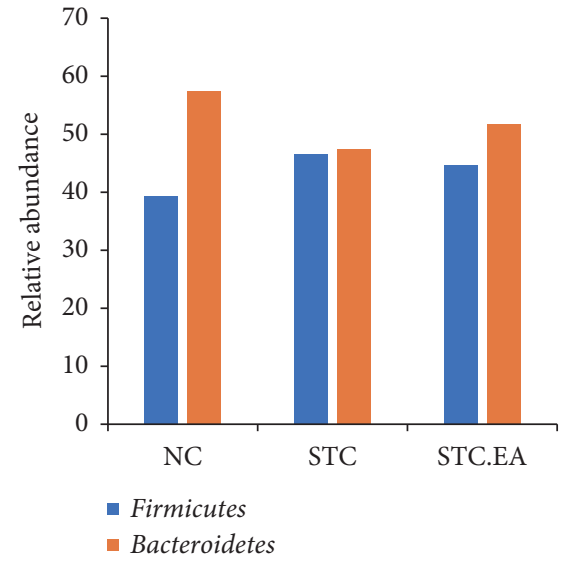

(b)

Figure 4: Effects of EA on the gut microbiota at the phylum level in constipated mice. (a) Pie charts at the phylum level. (b) The relative abundance of Firmicutes and Bacteroidetes.

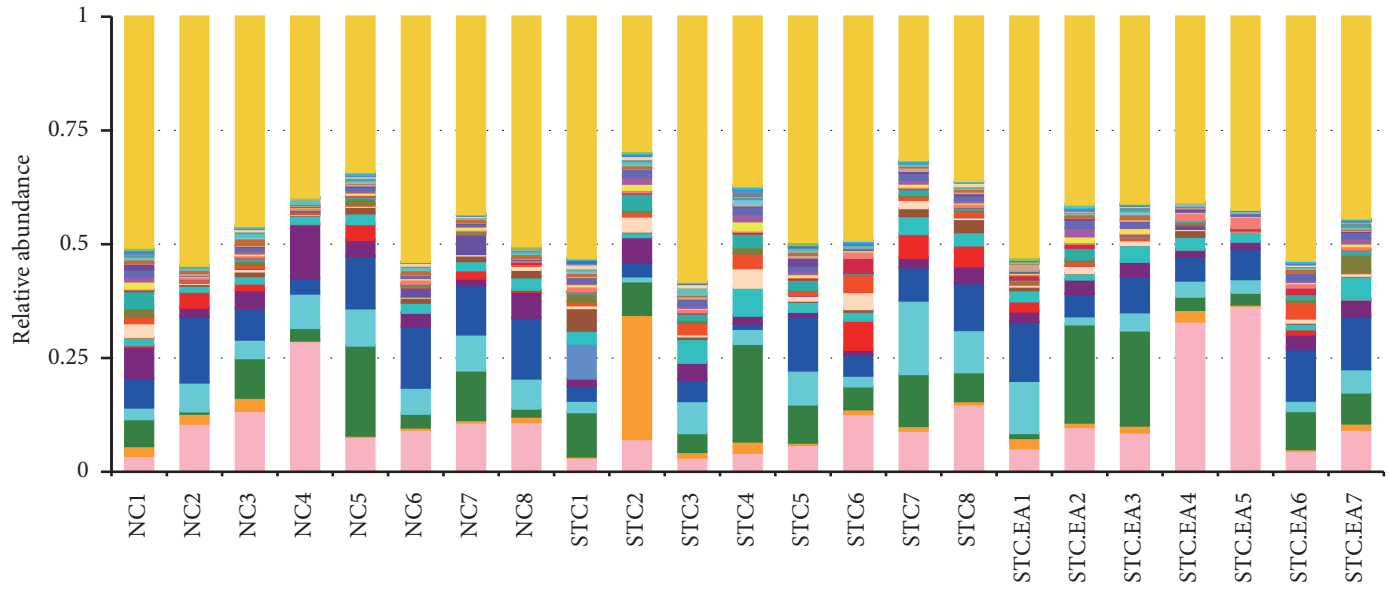

Sample name

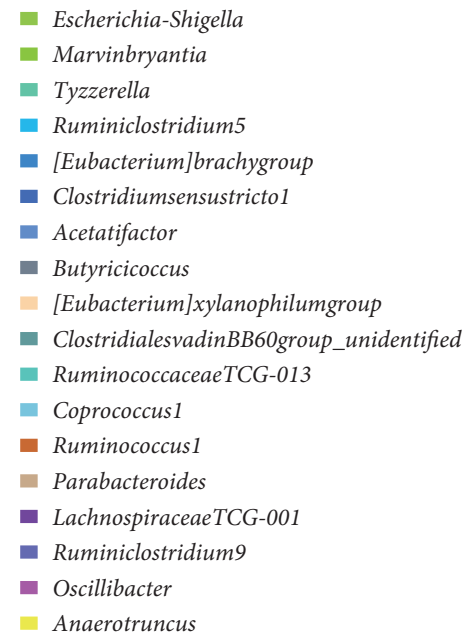

$$
\begin{aligned}
& \text { - Enterorhabdus } \\
& \text { - Desulfovibrio } \\
& \text { - Ruminiclostridium } \\
& \text { - Blautia } \\
& \text { - Lachnoclostridium } \\
& \text { - Akkermansia } \\
& \text { - Roseburia } \\
& \text { - PrevotellaceaeTCG-001 } \\
& \text { - Alistipes } \\
& \text { - } \text { Alloprevotella } \\
& \text { - Rumicibacter } \\
& \text { - Muribaculum } \\
& \text { Bacteroides } \\
& \text { - LachnospiraceaeNK4A136group } \\
& \text { - Helicobacter } \\
& \text { Lactobacillus }
\end{aligned}
$$

(a)

FIgURE 5: Continued. 


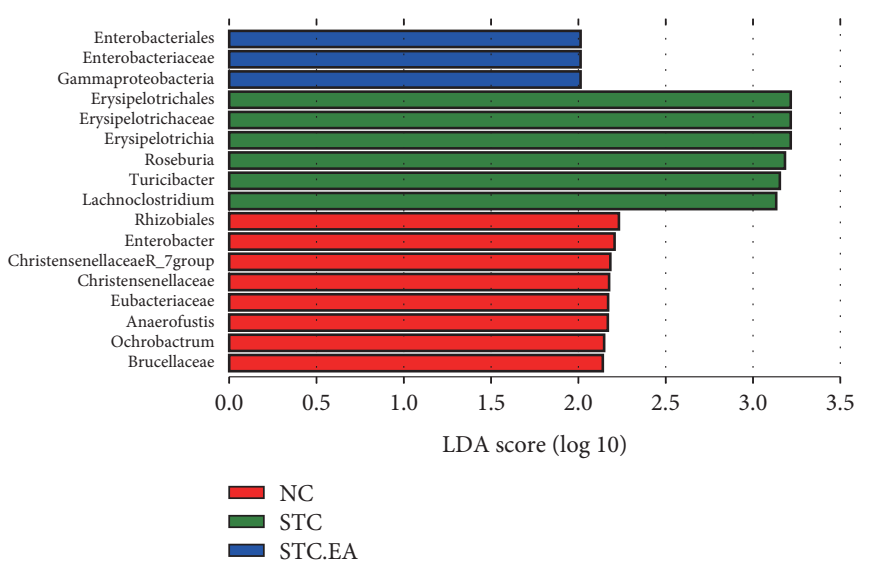

(b)

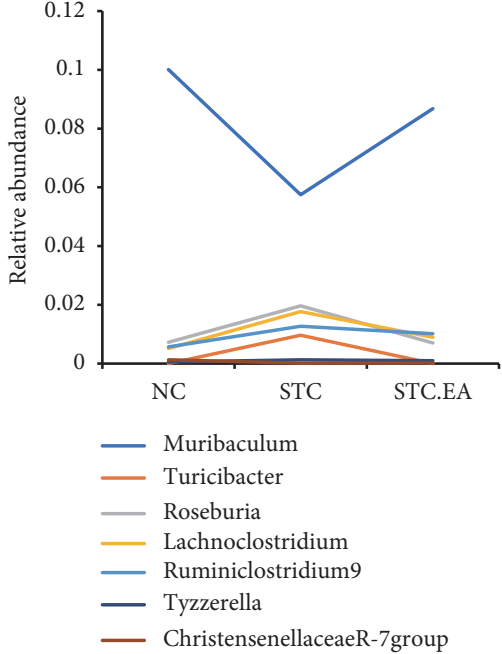

(c)

Figure 5: Effects of EA on gut microbiota at the genus level in constipated mice. (a) 35 most abundant genera in the three groups. (b) LEfSe analysis, $P=0.05$ for the Kruskal-Wallis test, LDA score $\geq 2.0$. (c) The relative abundance of the main altered microbes.

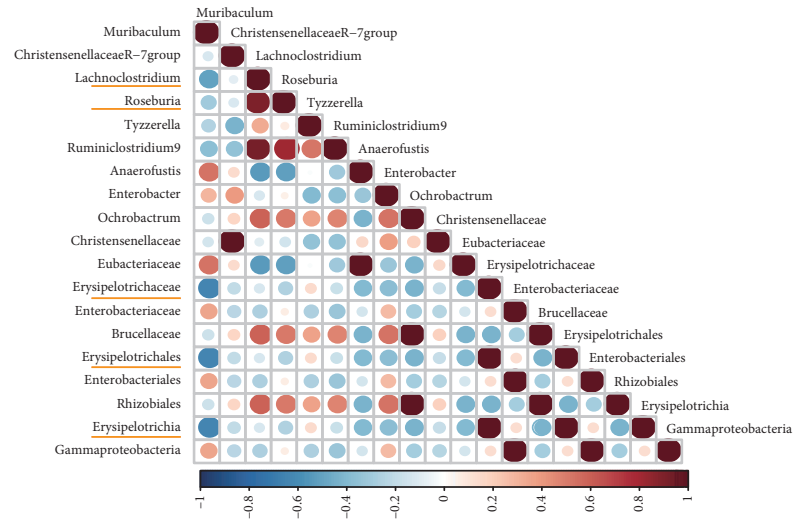

(a)

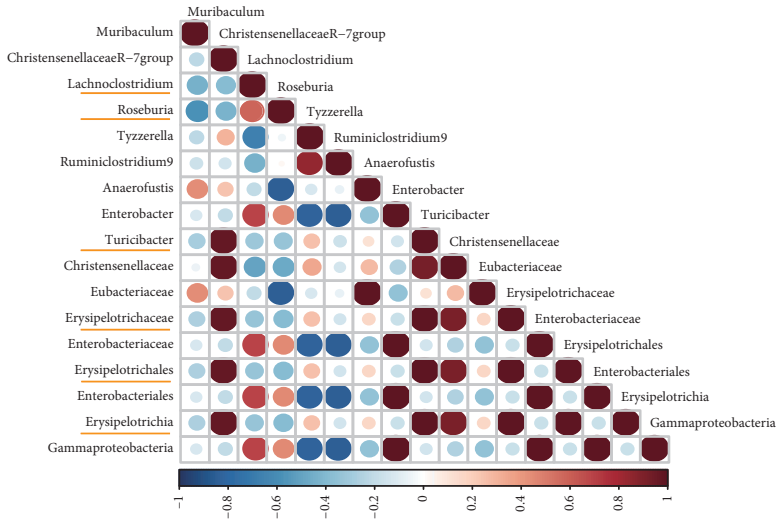

(b)

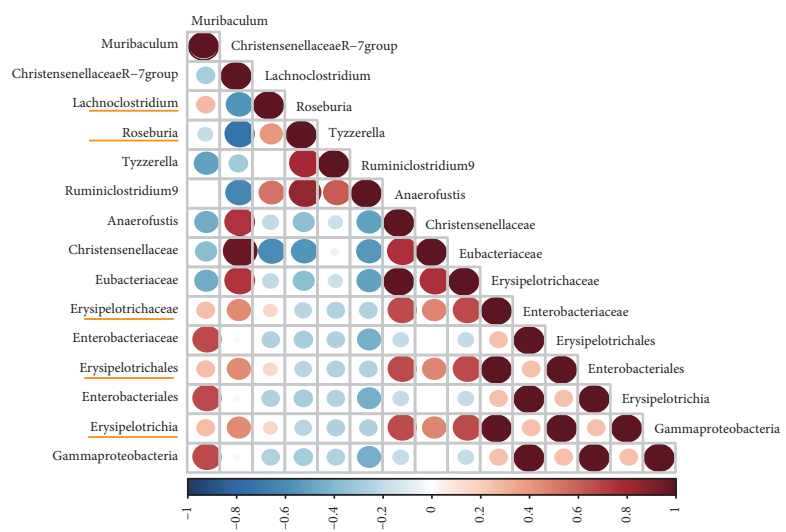

(c)

FiguRE 6: Relationship between certain microbes selected by LEfSe and metastat analysis. Red circles represent positive correlations; blue circles represent negative correlations. Darker shades and larger circles represent higher correlations. The microbes underlined in red were dominant in the STC group. (a) NC. (b) STC. (c) STC.EA. 


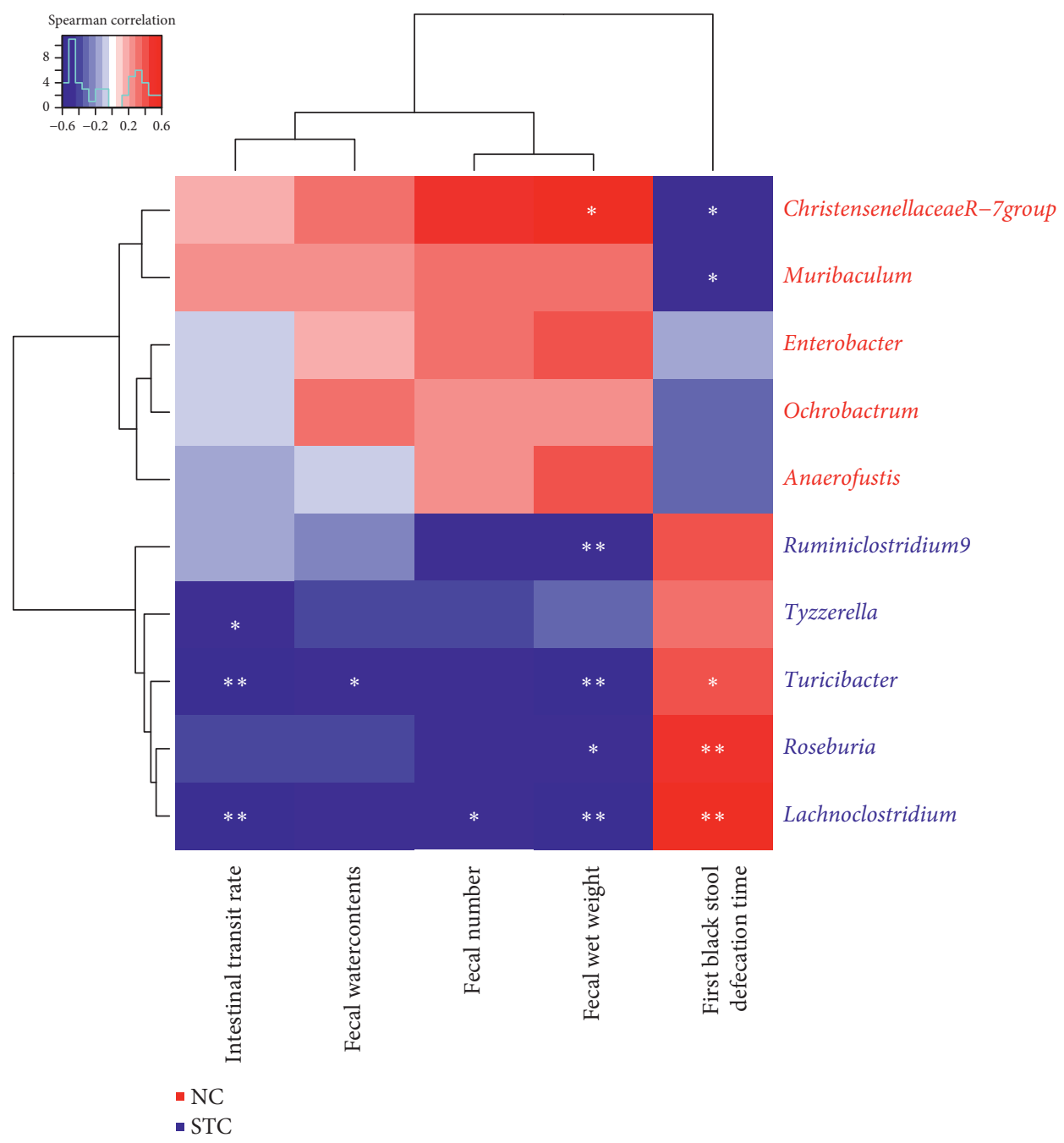

Figure 7: Correlation analysis between the main altered microbes at the genus level and constipation-related indices. Spearman correlation coefficients are represented from blue (negative correlation, -0.6) to red (positive correlation, 0.6). The microbes marked in red were dominant in the NC group, and microbes marked in blue were dominant in the STC group. ${ }^{*} P<0.05 ;{ }^{*}{ }^{*} P<0.01$.

ST25 is unclear, and how this results in a sustained effect on FC is also unknown. Therefore, the full range of mechanisms of EA action in FC still needs to be determined.

The complex gut microbiota colonizing the gastrointestinal tract plays important roles in modulating gastrointestinal functions such as metabolism, immunity, and motility. Disturbances in the gut microbiota are closely associated with functional intestinal diseases including constipation-type irritable bowel syndrome and FC [22]. Several clinical trials have shown that the microbiota composition in patients with constipation differs to that in healthy individuals [33, 40, 41]. Pseudo-germ-free mice receiving fecal microbiota from STC patients develop the typical features of constipation and decreased colonic muscle contractility [34]. Thus, alterations in the intestinal microbiota induced by constipation could further contribute to intestinal motility disorders, triggering a vicious cycle between motility dysfunction and dysbiosis. This also raises the possibility that intestinal dysbiosis may be a refractory cause of STC. However, the bacterial species causing constipation are still poorly characterized, with many discrepancies reported between studies. At the phylum level, Firmicutes and Bacteroidetes are the two highest abundance bacterial phyla in both mice and human, and the F/B ratio is considered to be a hallmark parameter of intestinal health. $\mathrm{Zhu}$ et al. and Mancabelli et al. found that the relative abundance of Bacteroidetes in FC patients was lower, while the relative abundance of Firmicutes was higher, than in healthy controls [40, 41]. Liu et al. also speculated that the symptoms of constipation induced by a nonisoflavone diet might be associated with an increase of Firmicutes and decrease in Bacteroidetes [30]. Here, the change in Firmicutes and Bacteroidetes in STC mice was similar to that previously reported, suggesting that an increasing F/B could be used as a microbial biomarker of constipation. Of note, we found that STC mice receiving EA showed a decrease in $\mathrm{F} / \mathrm{B}$ that was closer to healthy mice.

It has been elegantly demonstrated that the intestinal bacterial balance is critical for maintaining normal gut motility in germ-free (GF) and gnotobiotic animals [42, 43]. 
At the genus level, the main altered microbes further confirmed that EA treatment reversed the imbalance in gut microbiota in STC mice to normal. Among these altered microbes, Christensenellaceae R-7, Muribaculum, Ruminiclostridium 9, Tyzzerella, Turicibacter, Roseburia, and Lachnoclostridium were statistically correlated with constipation-related indices and so might participate in the underlying therapeutic mechanism of EA. In terms of their possible functions, Lachnoclostridium is a newly discovered genus with respect to digestive diseases that has been shown to be positively correlated with the tryptophan metabolic pathway in the colonic mucus [44] and might synthesize butyrate via the aminobutyrate/succinate pathway [45]. Roseburia can produce short-chain fatty acids (SCFAs), in particular butyrate, which might provide energy and nutrients for intestinal mucosal cells and promote colonic transit [33]. It has been reported that the abundance of Roseburia was significantly reduced in constipation [46, 47], inconsistent with our results. Ruminiclostridium 9 belongs to the Ruminococcaceae family, which are well-known for their butyrateproducing properties. It has been reported that Ruminococcaceae are related to decreased intestinal permeability and have an immunosuppressive effect $[48,49]$. These data suggest that Lachnoclostridium, Roseburia, and Ruminiclostridium 9 are beneficial for the intestinal environment through the synthesis of butyric acid and the suppression of intestinal inflammation. However, in our study, the abundance of Lachnoclostridium, Roseburia, and Ruminiclostridium 9 increased in the STC group and was reversed by EA treatment. We speculate that these microbes mainly participate in an imbalanced microbial steady-state and EA treatment promotes restoration of intestinal homeostasis.

Turicibacter has been shown to produce proinflammatory factors and exhibit a higher relative abundance in colitis [50,51] and type 2 diabetes mellitus [52]. Tyzzerella has been found at higher abundance in patients with cardiovascular diseases [53] and inflammatory diseases [54]. Muribaculum and Christensenellaceae $R-7$ belong to the Muribaculaceae and Christensenellaceae families, respectively, and are autochthonous and benign species. It has been reported that reductions in Muribaculaceae and Christensenellaceae could result in disorders associated with obesity and inflammation [55-57]. Here, harmful bacteria such as Turicibacter and Tyzzerella increased while beneficial bacteria such as Muribaculum and Christensenellaceae R-7 decreased in STC mice. Furthermore, EA treatment increased the abundance of beneficial bacteria and reduced the abundance of harmful bacteria, thereby improving the intestinal environment to maintain normal physiology helping to prevent other pathological changes induced by constipation. Furthermore, the microbe-microbe interaction analysis between groups further confirmed that EA treatment improved the relationship between microbes disrupted by constipation and maintained the dynamic equilibrium for intestinal microecology. Therefore, we conclude that the unique advantage of acupuncture in the treatment of constipation may be related to rebalancing the gut microbiota.

The findings of this study lay the foundation for further mechanistic and clinical research into the application of EA in patients with FC. Accumulating evidence indicates that the gut microbiota directly regulates gut motility via the enteric nervous system $[43,58]$ or indirectly through the gut immune system [59]. In order to more accurately clarify the potential mechanism underlying EA-mediated resolution of constipation by the gut microbiota, it would be useful to study microbial metabolites such as SCFAs and bile acid, intestinal neurotransmitters such as 5-hydroxytryptamine (5-HT), intestinal endocrine cells, and cytokine expression. In addition, future studies should explore whether EA improves constipation and promotes gut motility in the absence of an imbalance in the gut microbiota by using germ-free mice and/ or antibiotic-treated mice, which could further delineate the role of gut microbiota in the effect of acupuncture on constipation. Intriguingly, it has recently been reported that EA stimulation drives sympathetic pathways in a somatotopy- and intensity-dependent manner [60]. The vagal modulation of inflammatory responses is an important mechanism by which acupuncture exerts its therapeutic effect on internal organ disorders [61]. Thus, we hypothesize that EA-induced changes in the gut microbial profile were probably attributable to somatosensory autonomic pathways.

\section{Conclusion}

This study showed that EA reversed the typical symptoms of constipation, compromised gastrointestinal motility, and intestinal dysbiosis seen in diphenoxylate-induced constipated mice. EA treatment effectively rebalanced the gut microbiota of constipated mice, especially by decreasing the Firmicutes/Bacteroidetes ratio. The association between EA and the gut microbiota represents a possible new mechanism by which EA promotes gastrointestinal motility and alleviates constipation. However, the exact mechanism by which EA modulates the gut microbiota requires further clarification. Future studies should explore whether gut microbiota recovery is a key target for EA in constipation or simply a response to the normal intestinal function induced by EA, as well as how EA regulates the ENS to promote gut motility by rebalancing gut microbiota.

\section{Data Availability}

The datasets used and analyzed during the current study are available from the corresponding author upon request.

\section{Ethical Approval}

All experimental procedures involving mice were in strict accordance with the animal welfare principles and were approved by the Animal Ethics Committee of Chengdu Chinese Medicine University (approval number: 2018-12).

\section{Conflicts of Interest}

The authors declare that they have no conflicts of interest.

\section{Authors' Contributions}

Mingmin $\mathrm{Xu}, \mathrm{Lu}$ Wang, and Yu Guo contributed equally to this study. 


\section{Acknowledgments}

The authors acknowledge Dr. Xiumei Feng from Guanghan People's Hospital of Sichuan Province. This study was supported by grants from the National Natural Science Foundation of China (81774430 and 82074554).

\section{Supplementary Materials}

Figure S1: representative photomicrographs of hematoxylin and eosin- (HE-) stained colon tissues. Figure S2: $\alpha$-diversity metrics of the three groups. (A) Observed species. (B) Chao 1. (C) Shannon index. (D) PD whole tree. Table S1: average abundance of significantly altered microbes at the genus level among the groups. (Supplementary Materials)

\section{References}

[1] J. W. Wiley and L. Chang, "Functional bowel disorders," Gastroenterology, vol. 155, no. 1, pp. 1-4, 2018.

[2] F. Mearin, C. Ciriza, M. Mínguez et al., "Clinical practice guideline: irritable bowel syndrome with constipation and functional constipation in the adult," Revista Española de Enfermedades Digestivas, vol. 108, pp. 332-363, 2016.

[3] O. S. Palsson, W. Whitehead, H. Törnblom, A. D. Sperber, and M. Simren, "Prevalence of Rome IV functional bowel disorders among adults in the United States, Canada, and the United Kingdom," Gastroenterology, vol. 158, pp. 1263-1273, 2020.

[4] S. X. Sun, M. Dibonaventura, F. W. Purayidathil, J.-S. Wagner, O. Dabbous, and R. Mody, "Impact of chronic constipation on health-related quality of life, work productivity, and healthcare resource use: an analysis of the National Health and Wellness Survey," Digestive Diseases and Sciences, vol. 56, no. 9, pp. 2688-2695, 2011.

[5] A. Sharma and S. Rao, "Constipation: pathophysiology and current therapeutic approaches," Gastrointestinal Pharmacology, vol. 239, pp. 59-74, 2017.

[6] K. Sumida, M. Z. Molnar, P. K. Potukuchi et al., "Constipation and risk of death and cardiovascular events," Atherosclerosis, vol. 281, pp. 114-120, 2019.

[7] A. F. Peery, S. D. Crockett, C. C. Murphy et al., "Burden and cost of gastrointestinal, liver, and pancreatic diseases in the United States: update 2018," Gastroenterology, vol. 156, no. 1, pp. 254-272, 2019.

[8] C. J. Black and A. C. Ford, "Chronic idiopathic constipation in adults: epidemiology, pathophysiology, diagnosis and clinical management," Medical Journal of Australia, vol. 209, no. 2, pp. 86-91, 2018.

[9] J. F. Johanson and J. Kralstein, "Chronic constipation: a survey of the patient perspective," Alimentary Pharmacology \& Therapeutics, vol. 25, no. 5, pp. 599-608, 2007.

[10] S. Birch, "Historical and clinical perspectives onDe Qi: exposing limitations in the scientific study of De Qi," The Journal of Alternative and Complementary Medicine, vol. 21, no. 1, pp. 1-7, 2015.

[11] J. D. Z. Chen, M. Ni, and J. Yin, "Electroacupuncture treatments for gut motility disorders," Neurogastroenterology \& Motility, vol. 30, Article ID e13393, 2018.

[12] L. Pei, H. Geng, J. Guo et al., "Effect of acupuncture in patients with irritable bowel syndrome: a randomized controlled trial," Mayo Clinic Proceedings, vol. 95, no. 8, pp. 1671-1683, 2020.
[13] D. Horta, A. Lira, M. Sanchez-Lloansi et al., "A prospective pilot randomized study: electroacupuncture vs. Sham procedure for the treatment of fatigue in patients with quiescent inflammatory bowel disease," Inflammatory Bowel Diseases, vol. 26, no. 3, pp. 484-492, 2020.

[14] Z. Liu, Y. Liu, H. Xu et al., "Effect of electroacupuncture on urinary leakage among women with stress urinary incontinence: a randomized clinical trial," Jama, vol. 317, no. 24, pp. 2493-2501, 2017.

[15] L. Zhao, J. Chen, Y. Li et al., "The long-term effect of acupuncture for migraine prophylaxis: a randomized clinical trial," Jama Internal Medicine, vol. 177, no. 4, pp. 508-515, 2017.

[16] S. N. Garland, S. X. Xie, K. DuHamel et al., "Acupuncture versus cognitive behavioral therapy for insomnia in cancer survivors: a randomized clinical trial," JNCI: Journal of the National Cancer Institute, vol. 111, no. 12, pp. 1323-1331, 2019.

[17] J.-W. Yang, L.-Q. Wang, X. Zou et al., "Effect of acupuncture for postprandial distress syndrome: a randomized clinical trial," Annals of Internal Medicine, vol. 172, no. 12, pp. 777-785, 2020.

[18] Z. Liu, S. Yan, J. Wu et al., "Acupuncture for chronic severe functional constipation: a randomized trial," Annals of Internal Medicine, vol. 165, no. 11, pp. 761-769, 2016.

[19] H. Zheng, Z. S. Liu, W. Zhang et al., "Acupuncture for patients with chronic functional constipation: a randomized controlled trial," Neurogastroenterology \& Motility, vol. 30, Article ID e13307, 2018.

[20] P. Vitellio, G. Celano, L. Bonfrate, M. Gobbetti, P. Portincasa, and M. De Angelis, "Effects of bifidobacterium longum and lactobacillus rhamnosus on gut microbiota in patients with lactose intolerance and persisting functional gastrointestinal symptoms: a randomised, double-blind, cross-over study," Nutrients, vol. 11, no. 4, p. 886, 2019.

[21] X. Zhang, H. Tian, L. Gu et al., "Long-term follow-up of the effects of fecal microbiota transplantation in combination with soluble dietary fiber as a therapeutic regimen in slow transit constipation," Science China Life Sciences, vol. 61, no. 7, pp. 779-786, 2018.

[22] T. Ohkusa, S. Koido, Y. Nishikawa, and N. Sato, "Gut microbiota and chronic constipation: a review and update," Frontiers in Medicine, vol. 6, p. 19, 2019.

[23] D. O. V. S. Gomes and M. B. d. Morais, "Gut microbiota and the use of probiotics in constipation in children and adolescents: systematic review," Revista Paulista de Pediatria, vol. 38, Article ID e2018123, 2020.

[24] H. Wang, Q. Wang, C. Liang et al., “Acupuncture regulating gut microbiota in abdominal obese rats induced by high-fat diet," Evidence-Based Complementary and Alternative Medicine, vol. 2019, Article ID 4958294, 12 pages, 2019.

[25] X. Xu, X. Feng, M. He et al., "The effect of acupuncture on tumor growth and gut microbiota in mice inoculated with osteosarcoma cells," Chinese Medicine, vol. 15, p. 33, 2020.

[26] R. Wang, J. Sun, G. Li et al., "Effect of subsp. MN-Gup on constipation and the composition of gut microbiota," Beneficial Microbes, vol. 14, pp. 1-12, 2020.

[27] Y. Zhan, X. Tang, H. Xu, and S. Tang, "Maren pills improve constipation via regulating AQP3 and NF-B signaling pathway in slow transit constipation in vitro and in vivo," Evidence-Based Complementary and Alternative Medicine, vol. 2020, Article ID 9837384, 12 pages, 2020.

[28] S. Liu, D. Sui, W. Fu et al., "Laxative effects of yangyin tongmi capsule on a model of diphenoxylate-induced constipation in 
mice," Evidence-Based Complementary and Alternative Medicine, vol. 2020, Article ID 1471824, 9 pages, 2020.

[29] S. Yan, Y.-Z. Yue, X.-P. Wang et al., "Aqueous extracts of promoted intestinal motility in loperamide-induced constipation rats by ameliorating the interstitial cells of cajal," Evidence-Based Complementary and Alternative Medicine, vol. 2017, Article ID 6236904, 13 pages, 2017.

[30] J. Liu, R. Chang, X. Zhang, Z. Wang, J. Wen, and T. Zhou, "Non-isoflavones diet incurred metabolic modifications induced by constipation in rats via targeting gut microbiota," Frontiers in Microbiology, vol. 9, p. 3002, 2018.

[31] J. R. White, N. Nagarajan, and M. Pop, "Statistical methods for detecting differentially abundant features in clinical metagenomic samples," PLoS Computational Biology, vol. 5, Article ID e1000352, 2009.

[32] Q. He, C. Han, L. Huang et al., "Astragaloside IV alleviates mouse slow transit constipation by modulating gut microbiota profile and promoting butyric acid generation," Journal of Cellular and Molecular Medicine, vol. 24, no. 16, pp. 9349-9361, 2020.

[33] G. Parthasarathy, J. Chen, X. Chen et al., "Relationship between microbiota of the colonic mucosa vs feces and symptoms, colonic transit, and methane production in female patients with chronic constipation," Gastroenterology, vol. 150, no. 2, pp. 367-379, 2016.

[34] X. Ge, W. Zhao, C. Ding et al., "Potential role of fecal microbiota from patients with slow transit constipation in the regulation of gastrointestinal motility," Scientific Reports, vol. 7, p. 441, 2017.

[35] C. Liang, K. Wang, B. Xu, and Z. Yu, "Electroacupuncture at acupoint ST 37(Shangjuxu) improves function of the enteric nervous system in a novel mouse constipation model," BMC Complementary and Alternative Medicine, vol. 16, p. 392, 2016.

[36] C. Liang, K. Y. Wang, M. R. Gong, Q. Li, Z. Yu, and B. Xu, "Electro-acupuncture at ST37 and ST25 induce different effects on colonic motility via the enteric nervous system by affecting excitatory and inhibitory neurons," Neurogastroenterology \& Motility, vol. 30, Article ID e13318, 2018.

[37] X. Zhu, Z. Liu, H. Qu et al., "The effect and mechanism of electroacupuncture at LI11 and ST37 on constipation in a rat model," Acupuncture in Medicine, vol. 34, no. 3, pp. 194-200, 2016.

[38] E. Noguchi, "Acupuncture regulates gut motility and secretion via nerve reflexes," Autonomic Neuroscience, vol. 156, no. 1-2, pp. 15-18, 2010.

[39] A. Sato, Y. Sato, A. Suzuki, and S. Uchida, "Neural mechanisms of the reflex inhibition and excitation of gastric motility elicited by acupuncture-like stimulation in anesthetized rats," Neuroscience Research, vol. 18, no. 1, pp. 53-62, 1993.

[40] L. Zhu, W. Liu, R. Alkhouri et al., "Structural changes in the gut microbiome of constipated patients," Physiological Genomics, vol. 46, no. 18, pp. 679-686, 2014.

[41] L. Mancabelli, C. Milani, G. A. Lugli et al., "Unveiling the gut microbiota composition and functionality associated with constipation through metagenomic analyses," Scientific Reports, vol. 7, p. 9879, 2017.

[42] E. Husebye, P. M. Hellström, F. Sundler, J. Chen, and T. Midtvedt, "Influence of microbial species on small intestinal myoelectric activity and transit in germ-free rats," American Journal of Physiology-Gastrointestinal and Liver Physiology, vol. 280, no. 3, pp. G368-G380, 2001.
[43] P. C. Kashyap, A. Marcobal, L. K. Ursell et al., "Complex interactions among diet, gastrointestinal transit, and gut microbiota in humanized mice," Gastroenterology, vol. 144, no. 5, pp. 967-977, 2013.

[44] S.-B. Haange, N. Jehmlich, M. Hoffmann et al., "Disease development is accompanied by changes in bacterial protein abundance and functions in a refined model of dextran sulfate sodium (DSS)-Induced colitis," Journal of Proteome Research, vol. 18, no. 4, pp. 1774-1786, 2019.

[45] M. Vital, A. C. Howe, and J. M. Tiedje, "Revealing the bacterial butyrate synthesis pathways by analyzing (meta)genomic data," mBio, vol. 5, Article ID e00889, 2014.

[46] M. Zhuang, W. Shang, Q. Ma, P. Strappe, and Z. Zhou, "Abundance of probiotics and butyrate-production microbiome manages constipation via short-chain fatty acids production and hormones secretion," Molecular Nutrition \& Food Research, vol. 63, Article ID e1801187, 2019.

[47] D. R. Yarullina, M. U. Shafigullin, K. A. Sakulin et al., "Characterization of gut contractility and microbiota in patients with severe chronic constipation," PLoS One, vol. 15, Article ID e0235985, 2020.

[48] W. Wu, L. Lv, D. Shi et al., "Protective effect of against immune-mediated liver injury in a mouse model," Frontiers in Microbiology, vol. 8, p. 1804, 2017.

[49] S. Leclercq, S. Matamoros, P. D. Cani et al., "Intestinal permeability, gut-bacterial dysbiosis, and behavioral markers of alcohol-dependence severity," Proceedings of the National Academy of Sciences, vol. 111, no. 42, pp. E4485-E4493, 2014.

[50] A. Liu, H. Lv, H. Wang, H. Yang, Y. Li, and J. Qian, “Aging increases the severity of colitis and the related changes to the gut barrier and gut microbiota in humans and mice," The Journals of Gerontology: Series A, vol. 75, no. 7, pp. 1284-1292, 2020.

[51] Y.-N. Liang, J.-G. Yu, D.-B. Zhang et al., "Indigo naturalis ameliorates dextran sulfate sodium-induced colitis in mice by modulating the intestinal microbiota community," Molecules, vol. 24, no. 22, p. 4086, 2019.

[52] J. Zhao, Y. Li, M. Sun et al., "The Chinese herbal formula shenzhu tiaopi granule results in metabolic improvement in type 2 diabetic rats by modulating the gut microbiota," Evidence-Based Complementary and Alternative Medicine, vol. 201914 pages, Article ID 6976394, 2019.

[53] T. N. Kelly, L. A. Bazzano, N. J. Ajami et al., "Gut microbiome associates with lifetime cardiovascular disease risk profile Among bogalusa heart study participants," Circulation Research, vol. 119, no. 8, pp. 956-964, 2016.

[54] M. Olaisen, A. Flatberg, A. v. B. Granlund et al., "Bacterial mucosa-associated microbiome in inflamed and proximal noninflamed ileum of patients with crohn's disease," Inflammatory Bowel Diseases, vol. 27, no. 1, pp. 12-24, 2021.

[55] M. Do, E. Lee, M.-J. Oh, Y. Kim, and H.-Y. Park, "Highglucose or -fructose diet cause changes of the gut microbiota and metabolic disorders in mice without body weight change," Nutrients, vol. 10, no. 6, p. 761, 2018.

[56] Y. Liu, T. Li, A. Alim, D. Ren, Y. Zhao, and X. Yang, "Regulatory effects of stachyose on colonic and hepatic inflammation, gut microbiota dysbiosis, and peripheral CD4+ $\mathrm{T}$ cell distribution abnormality in high-fat diet-fed mice," Journal of Agricultural and Food Chemistry, vol. 67, no. 42, pp. 11665-11674, 2019. 
[57] L. Mancabelli, C. Milani, G. A. Lugli et al., "Identification of universal gut microbial biomarkers of common human intestinal diseases by meta-analysis," FEMS Microbiology Ecology, vol. 93, 2017.

[58] H. Cao, X. Liu, Y. An et al., "Dysbiosis contributes to chronic constipation development via regulation of serotonin transporter in the intestine," Scientific Reports, vol. 7, p. 10322, 2017.

[59] L. Grasa, L. Abecia, R. Forcén et al., "Antibiotic-induced depletion of murine microbiota induces mild inflammation and changes in toll-like receptor patterns and intestinal motility," Microbial Ecology, vol. 70, no. 3, pp. 835-848, 2015.

[60] S. Liu, Z.-F. Wang, Y.-S. Su et al., "Somatotopic organization and intensity dependence in driving distinct NPY-expressing sympathetic pathways by electroacupuncture," Neuron, vol. 108, no. 3, pp. 436-450, 2020.

[61] H.-D. Lim, M.-H. Kim, C.-Y. Lee, and U. Namgung, "Antiinflammatory effects of acupuncture stimulation via the vagus nerve," PLoS One, vol. 11, Article ID e0151882, 2016. 